\title{
Dans la conception des salles de régulation du SAMU, pouvoir communiquer est un enjeu de fiabilité de ses missions
}

When designing SAMU dispatch centers, the capacity to communicate is a crucial factor of mission reliability

Fabrice Bourgeois, Christophe Vanpoulle et Christine Ammirati

\section{OpenEdition Journals}

Édition électronique

URL : http://journals.openedition.org/activites/4516

DOI : 10.4000/activites. 4516

ISSN : $1765-2723$

Éditeur

ARPACT - Association Recherches et Pratiques sur les ACTivités

\section{Référence électronique}

Fabrice Bourgeois, Christophe Vanpoulle et Christine Ammirati, « Dans la conception des salles de régulation du SAMU, pouvoir communiquer est un enjeu de fiabilité de ses missions ", Activités [En ligne], 16-2 | 2019, mis en ligne le 15 octobre 2019, consulté le 17 octobre 2019. URL : http:// journals.openedition.org/activites/4516; DOI : 10.4000/activites.4516

Ce document a été généré automatiquement le 17 octobre 2019

\section{cc)}

Activités est mis à disposition selon les termes de la licence Creative Commons Attribution - Pas d'Utilisation Commerciale - Pas de Modification 4.0 International. 


\section{Dans la conception des salles de régulation du SAMU, pouvoir communiquer est un enjeu de fiabilité de ses missions}

When designing SAMU dispatch centers, the capacity to communicate is a crucial factor of mission reliability

Fabrice Bourgeois, Christophe Vanpoulle et Christine Ammirati

\section{NOTE DE L'ÉDITEUR}

Article soumis le 19 décembre 2018, accepté le 11 juin 2019

\section{NOTE DE L'AUTEUR}

Les auteurs souhaitent préciser que Perrine Pecot-Erzepa, ergonome, Raphaël Yharrassarry, ergonome du cabinet i ergo et Yves Hernot, acousticien ont contribué à l'intervention qui est rapportée dans l'article.

\section{Introduction}

1 La confiance dans les SAMU s'est trouvée sérieusement ébranlée, en 2018, suite à la mort d'une femme dont l'appel de détresse n'avait pas été pris au sérieux dans l'un d'entre eux. Tout le monde a pu entendre ou lire, sur les réseaux sociaux ou les médias, l'enregistrement de ses conversations avec une opératrice des sapeurs-pompiers puis une Assistante de Régulation Médicale (ARM). Il en est ressorti que la prise en charge avait été inadaptée. Bien évidemment et habituellement, c'est le médecin régulateur 
qui écoute l'appelant et lui donne une réponse après que l'ARM lui ait communiqué les informations systématiques collectées lors la prise en charge initiale de l'appel. Dans ce drame, l'enquête de l'Inspection Générale des Affaires Sociales ${ }^{1}$ remarque que la victime n'a pas « bénéficié d'une prise en charge conforme aux bonnes pratiques ». En faisant référence à la "conformité aux bonnes pratiques", on ne saurait mieux introduire le propos en constatant, effectivement, que l'organisation actuelle des SAMU résulte d'une construction empirique.

2 L'appel au 15 a été mis en place en 1979. Il faut attendre une loi de 1986 pour trouver la notion de détermination et déclenchement de la réponse la mieux adaptée, dans le délai le plus rapide qui définit un sens commun à tous les SAMU. Ceux-ci ont alors expérimenté et éprouvé des fonctionnements et des organisations correspondantes à cet objectif. Le caractère homogène de leur évolution doit notamment beaucoup aux expériences communes de prise en charge des situations de crise sanitaires pour lesquels ils se sont trouvés en première ligne (cf. la grève des généralistes, les épisodes de syndrome respiratoire aigu sévère au début des années 2000).

3 L'activité des salles de régulation du SAMU est toute orientée pour réaliser la régulation médicale, c'est-à-dire le processus qui comprend la demande de soins via l'appel, par nature non programmée, son traitement, la réponse à la demande et le déclenchement de l'intervention. Cette régulation nécessite, si l'appelant est le patient lui-même ou un tiers, un dispositif permettant le plus rapidement possible la reconstitution des informations sémiologiques et contextuelles nécessaires pour prendre une décision médicale d'orientation et de suivi du patient. Cependant, l'uniformisation des protocoles s'est structurée assez récemment, en 2011 lorsque la Haute Autorité de Santé (HAS) édite les modalités de prise en charge d'un appel de soins non programmé ${ }^{2}$. Ce document énonce des définitions fondatrices et structurantes (on y trouve les étapes de la prise en charge d'un appel ainsi que les missions de l'ARM et du médecin régulateur au regard de ces étapes et des éléments de fonctionnement), tout en prévenant qu'il s'agit de "recommandations de bonnes pratiques». Ainsi, il y est écrit que tout ARM doit avoir reçu une formation spécifique. Mais, la plupart du temps dans la réalité, celle-ci s'apparente à un compagnonnage dont il n'est pas garanti qu'il s'effectue de façon semblable d'un SAMU à l'autre ${ }^{3}$. Les textes réglementaires prévoient en effet une «formation d'adaptation à l'emploi » après la réussite d'un concours hospitalier sans évoquer la nécessité d'une formation initiale. Pour suppléer à cette carence dénoncée de longue date, un «référentiel métier» a été édité par les associations professionnelles en 2016 sous l'égide de la Société Française de Médecine d'Urgence.

4 En fait, les pratiques professionnelles d'un ARM, comme celle d'un médecin régulateur d'urgences, d'un médecin régulateur généraliste et d'un coordinateur ambulancier qui sont les métiers présents dans une salle de régulation, se construisent aussi en référence aux modalités de fonctionnement et de management propre à son SAMU, aux formes de coopération qui peuvent s'y développer ou pas, à la conception des moyens et espaces de travail dans leur capacité à favoriser ou pas la supervision, le contrôle, la confiance, le soutien...

5 L'accroissement régulier du nombre d'appels ( $+10 \%$ par an) et les caractéristiques des contextes sociaux et économiques territoriaux (notamment avec la désertification des médecins...) impactent également sur le fonctionnement des SAMU et leur capacité à maintenir, dans la durée, leur efficacité avec des moyens plus ou moins constants (Bertrand, Ammirati, Lecarpentier, Boyer, \& Marty, 2018). 
6 Le modèle de fonctionnement du SAMU fait face à une crise de croissance. Si l'amélioration de la formation de ses métiers est une affaire quasi entendue, la question de son organisation et des moyens de son fonctionnement fait débat. Les moyens sontils suffisants et seulement mal exploités par une mauvaise organisation? Les moyens doivent-ils évoluer pour favoriser une organisation plus réactive et plus adaptée aux évolutions de la nature des demandes et de leur nombre?

7 Dans une perspective de contribution au débat, nous présentons une intervention ergonomique réalisée dans le cadre de la conception d'une salle de régulation qui montre comment les postulats de conception spatiale structurent ou peuvent, au contraire, altérer le modèle de régulation médicale, sa fiabilité et son efficacité.

\section{Contexte de l'intervention}

En 2016, l'ensemble des activités du CHU d'Amiens qui étaient auparavant réparties entre deux établissements au nord et au sud de la ville a été regroupé dans le nouveau CHU au sud de la ville. Le projet de regroupement, très imposant, a fait l'objet de consultation des chefs de service concernés par le déménagement. Dès 2010, le chef de pôle de médecine d'Urgence, localisée au nord, découvre les esquisses de la future salle de régulation du SAMU-Centre 15. Les principes d'organisation fonctionnelle et spatiale sont totalement en écart avec la pratique du service (voir Figure 1). L'agencement des postes proposé préfigure une séparation entre les assistants de régulation médicale (ARM) d'une part, et les médecins régulateurs d'urgences hospitaliers (REG U) et généralistes (REG V) d'autre part. En effet, l'organisation qu'ils connaissent et qu'ils vivent au quotidien favorise un fonctionnement collectif intégrateur des deux métiers, ARM et médecin régulateur. Le projet, par contre, en intégrant une distinction dans l'espace coopératif, réduit ou supprime les modalités actuelles de communications comportementales et verbales auxquelles ils ont aussi recours et menace l'efficacité du collectif.

Figure $1:$ Aménagements en vigueur et prévu.

Figure 1: Current and planned layouts

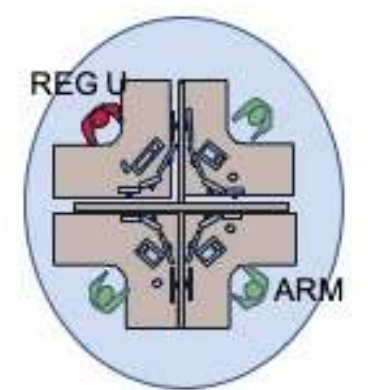

modèle d'aménagement utilisé par le SAMU avantle déménagement

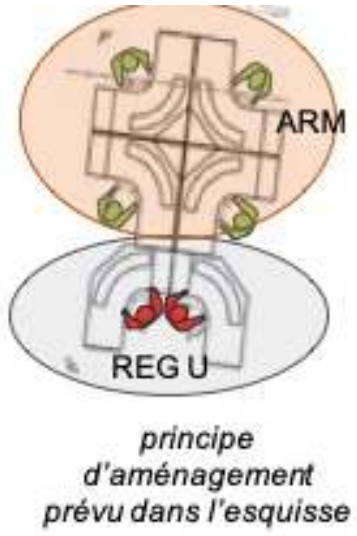

prévu dans l'esquisse

Cette esquisse inquiète le service, aussi bien son responsable que les médecins et les ARM. La proposition de nouvelle salle de régulation soutient un modèle de régulation médicale construit sur la seule transmission numérique vers le médecin des informations initiales que l'ARM collecte lors de la prise de l'appel. À l'origine de ce 
postulat, il y a l'idée que toutes autres formes de communication, notamment orales et gestuelles, sont considérées comme des troubles potentiels, des sources d'erreur susceptibles d'impacter la fiabilité du traitement de l'appel. Autrement dit, la fiabilité pourrait donc être totalement et exclusivement assurée par la transmission numérique des informations de l'ARM au médecin régulateur. La conception spatiale de l'esquisse vise donc à empêcher, du moins refroidir toute tentation de l'ARM d'interpeller et d'attirer l'attention du médecin par les gestes et la parole ou, inversement, toute tentation du médecin de se laisser interrompre et d'obtenir d'autres informations qui ne seraient pas transmises numériquement.

On devine clairement un choix décrit par Morvan, de Terssac et Peytavin (2000), visant à promouvoir une démarche d'Assurance Sécurité telle que celles existant dans les industries de procédés (nucléaire, chimie, pétrochimie) et les systèmes de transport (ferroviaire, aérien), et définies par des Autorités Réglementaires visant de manière explicite les « facteurs humains et organisationnels » susceptibles d'affecter la sécurité.

Ce postulat organisationnel à l'échelle du SAMU 80 prend le contre-pied de l'organisation actuelle qui, au contraire, considère que la communication numérique des informations initiales doit être complétée par d'autres formes de communications que les ARMs et les médecins régulateurs peuvent réaliser parce que la conception de leur espace de travail le permet. La conviction des acteurs du SAMU est que cela favorise une plus grande fiabilité de la régulation médicale et une plus grande garantie que les missions attendues de l'HAS soient réalisées.

Elle rejoint les conclusions de Marc et Amalberti (2002) en considérant que l'espace de régulation doit favoriser les interactions fondamentales dans le travail collectif en régulation médicale. Et celles de Benchekroun (2000) dans une intervention réalisée dans une autre salle de régulation des urgences, en attribuant une efficacité aux espaces de coopération proxémiques.

13 Pour argumenter en faveur d'un autre aménagement dans le futur CHU, le chef de service du SAMU d'Amiens obtient les crédits pour une intervention ergonomique (Vanpoulle, Bourgeois, Ammirati, Boyer, Amsallem, \& Decagny, 2016), afin de l'aider à :

- conforter le modèle d'efficacité des espaces de coopération proxémiques dans la salle de régulation actuelle ;

- établir une contre-proposition avec la participation des futurs utilisateurs à la conception.

Un cahier des charges doit être rendu au maitre d'ouvrage, avec les principes d'organisation spatiale favorisant l'écoute globale et les communications directes, les besoins de proximité et les principes d'aménagement, tout en anticipant l'augmentation probable de l'activité et l'évolution des moyens. L'intervention s'inscrit dans un phasage du projet encore intéressant pour transformer. Les marges de manœuvre de réimplantation restent importantes dans un cadre bâti certes intangible, mais spacieux. Le temps "devant soi » est contraint, mais laisse la possibilité d'organiser un processus participatif avec observations de l'activité, entretiens et groupes de travail. Seul point embarrassant, le maître d'ouvrage (MOA) nous évoque une limite calendaire très courte pour faire apparaitre l'emplacement des prises, des luminaires plafonds, des sorties de faisceaux au sol... avant même que nous ayons pu déterminer les principes d'aménagements des pôles. Ce MOA se trouve sous la pression du maître d'œuvre (MOE) prêt à pénaliser tout retard de livraison des cahiers des charges techniques, obligeant à accélérer le démarrage du phasage de notre propre intervention et à produire très rapidement des effets intangibles qui, heureusement, 
n'auront pas d'impacts déterminants sur les propositions finales. Il s'agit là d'une expérience concrète d'interférences et prise en compte des connaissances de l'activité des concepteurs au travers du projet d'intégrer les connaissances de l'activité des futurs utilisateurs (Falzon, 2005).

L'élaboration d'une nouvelle esquisse sera prise en main par un groupe de travail animé par les ergonomes et composé des métiers de la salle de régulation, des responsables du SAMU et des représentants du maître d'ouvrage qui étaient en contact direct avec le maître d'œuvre. Il a été instruit des différentes données du projet fournies par la maîtrise d'ouvrage et des résultats de notre analyse que nous présentons ci-dessous. Il a été le lieu de l'expression des points de vue des différents métiers et de l'élaboration des arbitrages d'aménagement. Notons que l'activité collective produite par l'intervention a pu trouver rapidement son efficacité dans la mesure où deux conditions étaient là réunies (Darses \& Falzon, 1994). D'abord des acteurs avaient tous une connaissance semblable de l'état de la situation (le problème, les enjeux, les hypothèses défendues ou contestées...), de sorte qu'il existait une forme de synchronisation sur le plan cognitif. Ensuite, ces mêmes acteurs partageaient un même niveau de connaissances (techniques, propriétés, procédures...), de sorte que nous avions une forme de synchronisation sur le plan de l'action (compréhension de la méthodologie, de contraintes calendaires...).

\section{Conforter la fiabilité des communications issues des espaces de coopérations proxémiques}

Dans les recommandations de bonne pratique de l'HAS, il est attendu de l'ARM, lors de la prise en charge initiale de l'appel, qu'il collecte des éléments non médicaux, le motif de recours, éventuellement des renseignements d'ordre général afin de les transmettre de façon précise et synthétique, et sans interprétation, au médecin régulateur. Le médecin régulateur prend le relais pour analyser ces premières informations qui doivent rendre compte $\mathrm{du}$ contexte ayant motivé l'appel.

17 L'efficacité de la relation entre l'ARM et le médecin régulateur est donc étroitement liée à la fiabilité du processus de "rendre compte de...» de la réalité de l'appel. Toutefois, cette réalité est soumise à une grande variabilité de situations qui va déterminer des contextes différents de communications et de transmissions d'informations. La revue de lecture de Leplat (2001) nous rappelle que le contexte s'invite, qu'on le veuille ou non pourrait-on rajouter, dans la gestion des communications. Dans l'activité de régulation des appels en urgence, le contexte de la relation entre l'appelant et le 15, d'une part, et au sein de la salle de régulation, d'autre part, change au sein d'une même journée ou au cours de la semaine selon les moments ou selon les circonstances locales et, plus généralement, selon les évolutions sociétales.

Ainsi, la reprise en main des acteurs du SAMU 80 est une façon de s'opposer au monde froid de la prescription organisationnelle et assurantielle dans lequel ils ne se reconnaissent pas (Bourgeois \& Hubault, 2013) et qui réfute des formes possibles d'autonomie individuelle et collective à l'intérieur d'un cadre qui fait sens pour tous et qui autorise des déformations et reformations pour tenir compte des "contextes" (Maggi, 1996). 
l'on souhaite le plus réactif et le plus rapide dans la prise de décisions. On peut supposer qu'un dilemme pour tout SAMU s'insinue dans la recherche du bon équilibre entre la nécessité de réduire la collecte de l'information à l'essentiel et la nécessité de prendre en compte le contexte de la relation. La réduction à l'essentiel permet de gagner du temps de traitement et d'être plus rapide dans la décision et l'intervention des secours. La préoccupation du contexte permet une meilleure qualité de la contribution de l'appelant qui y voit une compréhension du lien social qu'il projette en appelant le 15 et, en final, une plus grande fiabilité des informations collectées. Elle permet aussi une meilleure qualité des régulations entre les métiers de la salle de régulation par une meilleure transmission des éléments de contexte. Mais la prise en compte du contexte peut-elle représenter un élargissement couteux en temps autour de l'appel sur lequel se focalise l'organisation de la salle de régulation? ou bien peut-elle représenter au contraire une ressource profitable à un travail de qualité et, en conséquence, à la qualité de service?

Nous avons observé une vingtaine de systèmes de communications entre ARM et médecins régulateurs en prenant soin de les associer aux éléments de contexte de l'appel. Et bien évidemment, nous avons cherché à collecter une diversité de systèmes de communications à l'œuvre, notamment celui qui se rapproche le plus du modèle recommandé (transmission exclusive par voie numérique), mais aussi ceux qui s'en écartent en interférant d'autres modalités de communication (échange oral, déplacement, posture et geste particuliers...).

21 L'analyse des actions dans chacun de ses dispositifs de communication observés vise à savoir si ces «autres modalités de communication" résultent uniquement de la configuration de l'aménagement, sans qu'il y ait de valeur ajoutée notable, comme une tentation facile, mais sans intérêt? Ou bien résultent-elles d'une logique d'efficacité éprouvée et nécessaire dans des contextes bien identifiés, et qui serait facilitée par la configuration actuelle?

\subsection{Le cas d'un contexte d'appel urgent dont le traitement a pu être accéléré par des communications gestuelles et orales}

Dans cette observation de prise en charge initiale d'un appel, l'ARM a saisi les données et le médecin régulateur peut en disposer sur son écran (cf. dessin 1 de la Figure 2). Mais l'ARM se rend compte que le médecin ne prend pas «numériquement » la main sur cet appel.

Selon les circonstances (appelant rassurant, rassuré..., relative gravité...), l'ARM peut évaluer qu'une attente de la prise en charge par le médecin régulateur est acceptable. Dans le cas observé, le contexte ne s'y prête pas.

Ceci amène l'ARM à maintenir le contact téléphonique avec l'appelant. Elle se dresse sur son siège pour mieux voir le médecin afin d'interpréter son silence, mais aussi se manifester à ses yeux. Elle l'interpelle: «Bruno, tu es en ligne? ? (cf. dessin 2 de la Figure 2). Le médecin lui répond affirmativement. L'ARM attend quelques secondes en continuant de fixer le médecin. Celui-ci répète tout haut un numéro de téléphone qu'on lui communique (cf. dessin 3 de la Figure 2). Pour l'ARM, c'est une information sur le niveau d'engagement du médecin dans sa conversation en cours. 

Figure 2). L'ARM lui rend compte de son appel en attente «J'ai une dame de 28 ans qui aurait convulsé, elle a pris une plaquette de tramadol, elle n'est pas épileptique connue, elle s'est mise à vomir beaucoup, mais là elle cligne des yeux, sa mère est dans tous ses états... ils sont à Venonville... elle ne leur parle pas du tout... et elle est toute raide... » (cf. dessin 6 de la Figure 2). Le médecin lui dit que le tramadol n'est pas la seule explication, et qu'il faut envoyer le Service Mobile d'Urgence et de Réanimation (SMUR). L'ARM reprend le contact avec l'appelant et lui indique qu'on envoie le SMUR (cf. dessin 7 de la Figure 2).

Figure 2 : Attitudes significatives d'un processus de régulation (illustrations basées sur des photos).

Figure 2: Significant attitudes of a regulatory process (illustrations based on photos)

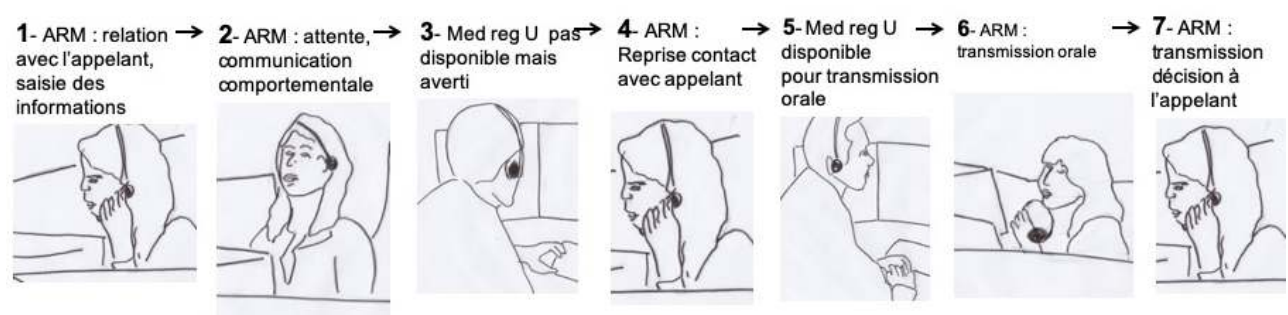
pratique se soit dessinée assez distinctement du prescrit. Le contexte s'est nourri des effets des actions entreprises :

- l'identification d'une transmission numérique qui n'est pas exploitée immédiatement par le médecin régulateur encore engagé dans l'appel précédent est un premier effet qui va déterminer un changement dans le processus ;

- l'ARM considère que la durée de cette attente a des conséquences pour une personne potentiellement en danger. Elle en est préoccupée. Elle prend alors l'initiative d'interpeller le médecin, d'abord en adoptant une posture décelable dans son champ de vision, ensuite à l'oral;

- Le médecin régulateur donne précocement à l'ARM des signes de la compréhension de son alerte.

Cette initiative signifie la nécessité d'un relais urgent dans l'analyse médicale et la réorganisation du prescrit. Elle s'inscrit dans des conventions entre ARM et médecins régulateurs, connues et acceptées, construites dans le temps et par le travail collectif (Caroly \& Barcellini, 2013).

En cela, cette "pratique " mobilise des canaux de communications ayant recours au langage verbal, mais aussi au langage des gestes et des attitudes du corps. Pour autant, ce recours n'a pas été distrayant, ni pénalisant du point de vue de la réactivité et rapidité de la réponse. Au contraire, ces "gestes-signes" associés à la parole 
constituent l'expressivité nécessaire (Leplat, 2013) qui permet de corriger une perte de fiabilité que l'application stricto sensu de la transmission numérique aurait générée. Notamment, ils ont évité un dépassement du temps de prise en charge comme le montre la figure 3. Sur cette figure, la partie droite représente le prescrit qui, en luimême, peut justifier la séparation physique des ARMs et des médecins régulateurs. Il est demandé à l'ARM de saisir les éléments non médicaux et les motifs et de les transmettre au médecin régulateur par voie numérique. L'affaire s'arrêterait là si l'économie de l'information avait été au rendez-vous et si aucun élément de contexte n'était venu contrarier l'efficacité attendue. La partie gauche représente la situation observée. La transmission orale s'est substituée à la lecture de la transmission écrite, comme forme de rattrapage de l'attente. En effet, dans cet exemple, le médecin se laisse résumer oralement les informations essentielles par l'ARM. Les estimant suffisantes, il lui communique sa décision oralement, ce processus permet d'éviter un temps de lecture des mêmes informations sur écran et un temps de prise en charge de l'appelant au téléphone.

Figure 3 : Comparaison entre processus prescrit et réalisé.

Figure 3: Comparison between prescribed and completed processes

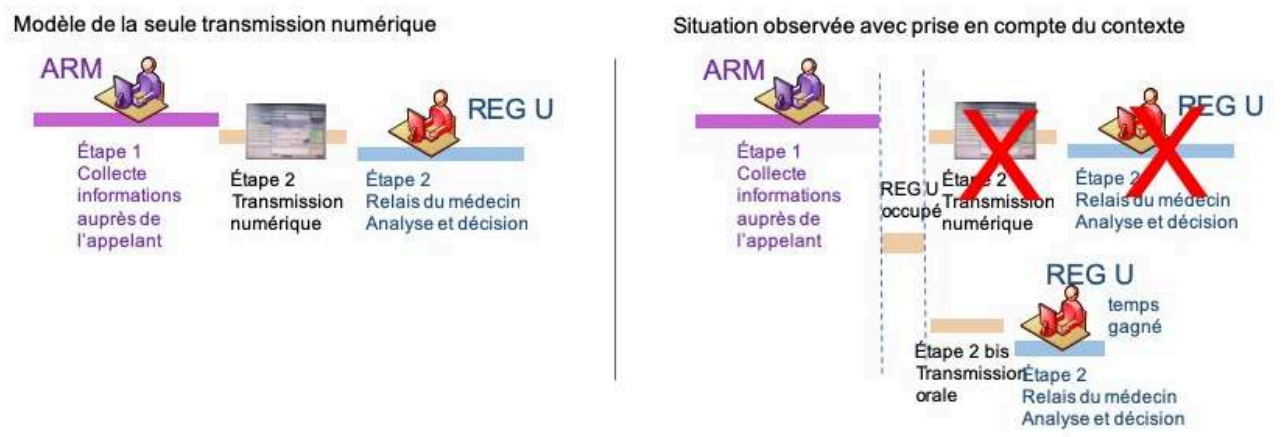

\subsection{Le cas d'un contexte d'appel potentiellement suspect, mais potentiellement urgent}

Dans cette observation, l'ARM a en ligne une personne qui téléphone du Pas de Calais pour signaler une urgence dans la Somme qui concernerait sa propre mère (cf. dessin 1 de la Figure 4). Elle indique à l'ARM que son petit garçon qui est avec sa grand-mère l'a appelée pour lui dire qu'elle est tombée et qu'elle ne va pas bien.

L'ARM a besoin d'avoir en ligne le petit garçon pour obtenir plus d'informations sur l'état de la grand-mère, mais aussi vérifier que l'appel n'est pas une farce. Elle avertit la mère « ... bon je rappelle sur place... je rappelle sur place... oui, je rappelle votre fils sur place ». En même temps, elle regarde une collègue ARM à proximité qui écoute la conversation. Elle exprime une mimique pour faire partager son besoin de vérifier la fiabilité de l'appel.

En attendant que l'appel vers le petit garçon décroche, l'ARM débute un résumé de l'affaire au médecin régulateur des urgences qui est disponible (cf. dessin 2 de la Figure 4) : « c'est compliqué, c'est le SAMU 62 qui me passe la fille qui habite à $100 \mathrm{~km}$ de là, qui me prévient que sa mère est tombée du lit et elle habite à... ». Le médecin prend des notes sur un papier. 

garçon : «allo bonjour Monsieur, est-ce que je peux parler au petit garçon qu'il y a chez vous s'il vous plait... parce que sa maman vient de m'appeler pour me dire qu'il était là, je voudrais lui parler s'il vous plait... ». Le Médecin s'adresse à un autre ARM, debout, qui écoute depuis le début : « là je ne comprends pas... qui est tombé, le petit garçon ou la mère ?» (cf. Figure 4 et dessin 3 de la figure 4). L'autre ARM répond « c'est la grandmère je crois, c'est le petit fils qui est près d'elle et il est allé chez un voisin pour donner l'alerte ». Le médecin commente : « ... oui, mais là on va parler à un enfant... ».

Le médecin écoute la conversation que l'ARM a avec l'enfant (cf. dessin 4 de la figure 4) : "Mathis, bonjour c'est le SAMU, je pense que c'est ta maman que j'ai eu au téléphone, elle m'a prévenue que ta mamie était tombée, c'est çà ? ... et elle te parle toujours ta mamie ? ... elle est toujours par terre ? ... ah tu l'as remise dans le lit... d'accord avec papi... et elle te parle mamie? ...». Le Med Reg U s'est déplacé (cf. dessin 5 de la Figure 4) derrière l'ARM et lit les informations saisies sur son écran (cf. dessin 6 de la Figure 4). L'ARM continue l'entretien avec l'enfant : "d'accord et là tout de suite si tu l'appelles, si tu cries mamie, elle va te répondre ?... » Le Med Reg U s'adresse à l'ARM à voix basse pour lui poser une question. L'ARM répond à voix basse. Puis le Med Reg U lui demande l'âge du petit garçon « ... toi tu as quel âge Mathis ?... tu as 11 ans... ». Le médecin fait un geste signifiant qu'il n'est pas nécessaire de continuer la demande d'information et indique à l'ARM sa décision. L'ARM dit à l'enfant : « on va t'envoyer les secours chez toi, une ambulance... ».

Figure 4 : Attitudes significatives d'un second processus de régulation (illustrations basées sur des photos).

Figure 4 : Significant attitudes of a second regulatory process (illustrations based on photo)

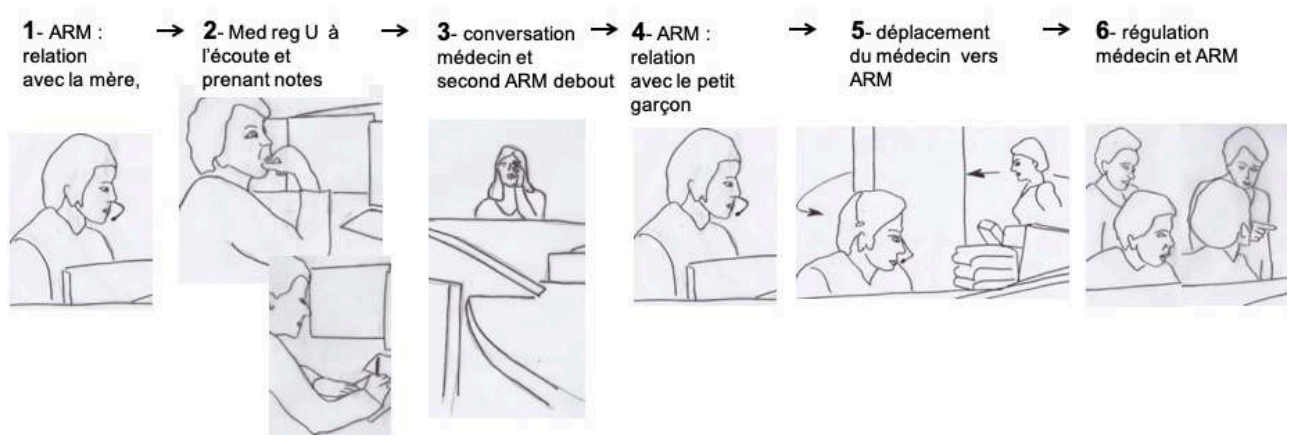

Plusieurs enjeux se croisent et vont se dénouer dans le déroulement de cette prise en charge. D'abord celui pour l'ARM de vérifier qu'il ne s'agit pas d'un canular dès lors que l'appelant téléphone pour une personne qui habite ailleurs. La confrontation avec le petit garçon doit alors permettre de le vérifier. Mais la seconde raison d'avoir le petit fils en ligne, dès lors qu'il ne s'agit plus d'un canular, est d'obtenir des informations plus récentes et plus précises de l'état de la grand-mère pour renseigner les données pertinentes à transmettre au médecin régulateur. Un autre enjeu que dévoile le médecin régulateur en se montrant disponible est celui de la pertinence des informations que peut donner un enfant de 11 ans. Le médecin considère que la décision d'envoyer une ambulance peut s'envisager sur une base d'informations moins complète, dès lors que celles disponibles lui semblent suffisantes. Pour accélérer la prise de connaissances, le médecin se déplace derrière le poste de l'ARM en train de saisir les données et les consulte sur son écran. C'est alors qu'il signifie à l'oreille de 
l'ARM qu'il en sait suffisamment pour décider d'envoyer une ambulance. L'ARM interrompt alors l'interrogation du petit garçon et l'avertit qu'on vient chez sa mamie.

Là s'est jouée une régulation entre deux orientations :

- celle que développe l'ARM qui vise à s'assurer de la fiabilité de l'appel et à actualiser auprès du petit garçon des informations initiales de «seconde main » (en provenance d'une mère non présente) pour saisir toutes les informations habituellement nécessaires au médecin régulateur;

- celle que développe le médecin régulateur qui juge sans valeur ajoutée la poursuite d'informations au-delà de celles déjà disponibles, à cause de l'âge du petit garçon et surtout du temps écoulé depuis la première prise en charge par le SAMU 62, le relais au SAMU 80, la ré-explication de la situation par la mère au SAMU 80, l'appel vers le petit fils...

Cette observation rend compte d'une re-construction collective du prescrit, là encore, comme dans le cas précédent, pour accélérer le processus de régulation médicale. Si le prescrit s'était imposé dans notre contexte, le médecin régulateur n'aurait pris sa décision qu'après avoir lu sur son écran les informations après leur collecte définitive par l'ARM et éventuellement, après une conversation avec la mère ou son petit-fils. La proximité physique a joué dans la captation précoce des éléments de conversation de l'ARM. Mais pas seulement, ce sont les conventions entre ARMs et médecins régulateurs, connues et acceptées par eux-mêmes, construites dans le temps et par le travail collectif. Ces règles communes ont permis à l'ARM d'obtenir l'écoute de son médecin régulateur lors de ses échanges avec les différents appelants et au médecin d'interférer dans les échanges pour en accélérer le dénouement.

Ce mode opératoire a mobilisé le langage de gestes et attitudes du corps particuliers (se redresser, se regarder, se mettre debout, se déplacer, se rapprocher...) et le langage oral qui visent à accélérer la transmission d'informations, partager avec un pair une perception, évaluer la pertinence de l'action en cours... Comme dans le cas précédent, il a su corriger une perte d'efficacité que l'application stricto sensu de la transmission numérique aurait générée.

Cette pratique a été probablement possible parce qu'aucun autre appel grave n'a nécessité la prise en charge par le médecin, rendu alors disponible.

\subsection{Le périmètre réel des communications mobilisées dans la régulation médicale}

41 Jusqu'à présent, notre focale sur le binôme ARM et médecin régulateur se justifie par l'évidente importance de la réussite qu'on attend du démarrage du processus de régulation : la réception de l'appel par l'ARM et son relais au médecin régulateur.

42 Les études de cas montrent que le processus de régulation est une prise en charge collective mobilisant un langage (Leyreloup, 2008) constitué de gestes, de postures, de mots, tous encadrés par des savoir-faire, des connaissances, des procédures et des règles éprouvés dans leur efficience par des coopérations précédentes et successives. Cette construction va même au-delà des seuls médecins et ARMs.

43 Comme le montre la figure 5, l'ensemble du processus de régulation s'organise au moyen de nombreuses autres communications avec les autres métiers présents dans la salle de régulation (médecin régulateur généraliste de ville, coordinateur ambulancier...) et les métiers en charge de l'intervention décidée par le médecin 
régulateur (médecins, hospitaliers ambulanciers, pédiatres, infirmiers, puéricultrices, pilotes d'hélicoptère...). Comme le montre cette même figure, l'ARM gère des communications avec le coordinateur ambulancier qui est chargé d'ordonner à un ambulancier privé un déplacement moins urgent mais aussi avec les intervenants du SAMU, par téléphone et radio, pour les interventions d'urgence (les ambulanciers, les composantes de l'équipe médicale qui intervient, le pilote d'hélicoptère le cas échéant). Le médecin régulateur est également concerné par ces interfaces.

Ces communications sont complémentaires au transfert des données numériques, pour insister sur des éléments de contexte qui semblent utiles de donner aux intervenants lors des départs.

Le contenu particulier de ces échanges se constitue également par les retours d'intervention que font les intervenants directement dans la salle de régulation lorsque le flux d'appels le permet.

Figure 5 : Flux et intensité des communications orales directes. Figure 5: Flow and intensity of direct oral communications

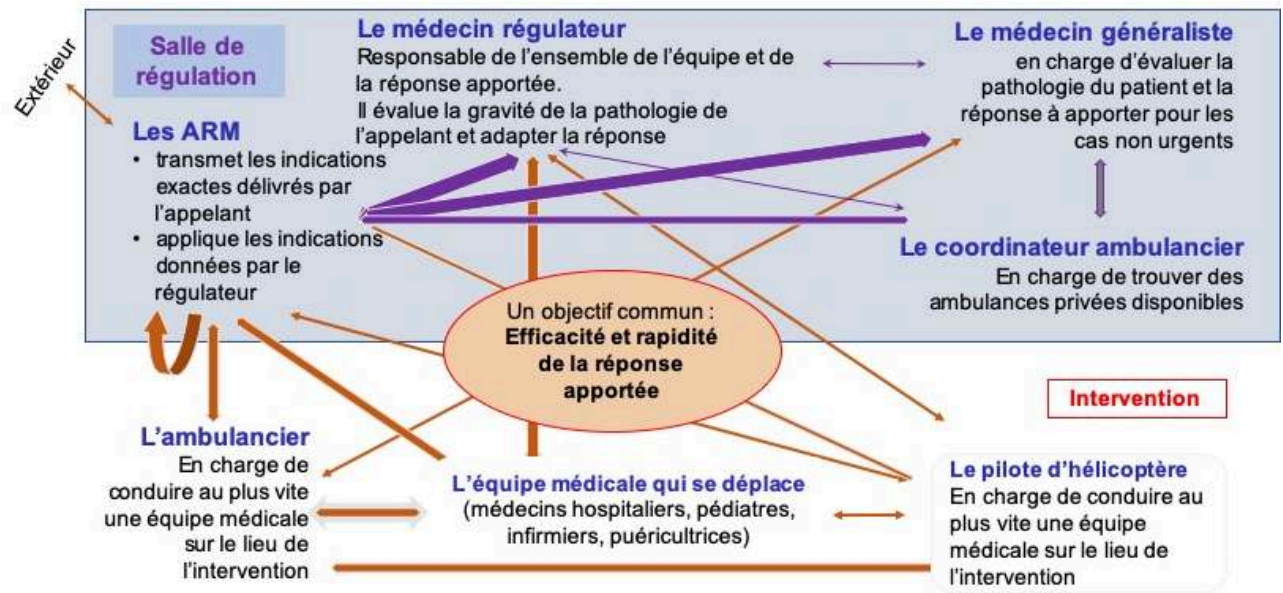

C'est particulièrement le cas lors des postes de nuit. Les ARMs et les médecins régulateurs apprécient ces visites qui ont plusieurs fonctions. Elles donnent des nouvelles de la prise en charge sur place et permettent à ceux qui ont été les premiers en contact avec les appelants de connaître une partie de dénouement, notamment quand le contenu de l'appel a pu être source de forte inquiétude et si cela concerne des enfants. Elles renseignent également sur la pertinence de l'analyse et de la décision, sur l'efficacité de sa rapidité, sur la qualité des informations collectées et transmises. Ce retour d'expérience fait système d'évaluation, d'amélioration et de reconnaissance. Il contribue à se mettre d'accord sur des manières de faire entre "ceux de la salle de régulation" et "ceux de l'intervention d'urgence». Les entretiens et observations mettront également en évidence l'importance pour les médecins régulateurs et les ARMs d'avoir une vue sur les départs et retours d'ambulances du SMUR afin de disposer d'une actualisation permanente des moyens mobiles disponibles (surveillance vidéo). Lorsque le flux d'appels ne permet pas ce retour et partage d'information, la salle de repas est un lieu d'échanges important.

47 Ces observations nous rapprochent de Mayen (2000) pour qui la dynamique des échanges conversationnels nécessite un système de règles qui régit les échanges 
langagiers entre humains, l'ajustement des univers de référence, la négociation des solutions entre les interlocuteurs.

\section{Décrire ce qui fait le pouvoir d'agir en salle de régulation et que la conception de l'espace de travail doit soutenir}

Les différentes observations réalisées ont permis de décrire des pratiques utilisatrices de plusieurs modalités de communication, selon le contexte et en accord avec des règles collectives connues et reconnues de tous. Elles sont productrices d'une meilleure fiabilité et efficacité que l'utilisation de la seule voie de communication numérique. Nous avons ainsi, sous nos yeux, un collectif de plusieurs métiers engagés dans un objectif commun de régulation médicale le plus rapide. Ce collectif met en œuvre une « organisation capacitante » (Oudet, 2012), c'est-à-dire :

- permettant de développer des «capacités» (cf. savoir-faire, pouvoir d'agir ${ }^{4} .$. ) en correspondance aux diverses situations contextuelles qui se présentent ;

- disposant d'un environnement favorable (proximité physique, agencement favorable aux échanges, construction de règles collectives, écoute et soutien des pairs, écoute et soutien entre médecin et ARM...) qui sont autant de possibilités (cf. «capabilités, pouvoir agir ${ }^{5}$ ) d'expérimenter, éprouver, améliorer... développer en quelque sorte des pratiques adaptées aux contextes et à leur évolution.

\subsection{Exemples de capacités devant être soutenues par un espace de coopération proxémique}

Notre analyse précédente des pratiques de prise en charge des appels nous a permis d'établir des motifs du rapport de proximité recherché et mis en pratique par les occupants de la salle de régulation. Ils ont été formalisés et validés en groupe de travail. Les figures 7 à 10 rendent compte des capacités et des ressources que chaque métier développe et mobilise dans les différents contextes de prise en charge.

\subsubsection{Les raisons d'un rapport de proximité du médecin régulateur avec les autres métiers de la salle de régulation}

Par exemple, la figure 6 montre que la capacité du médecin régulateur d'urgence est augmentée s'il peut écouter la conversation de l'ARM avec un appelant. Cette capacité d'écoute lui permet d'anticiper l'information avant qu'elle lui soit livrée numériquement et d'anticiper les questions qu'il lui posera. Ce gain de temps est jugé inestimable pour un départ plus précoce de l'ambulance. 
Figure 6 : Médecin Régulateur d'Urgence face aux ARM.

Figure 6: Emergency Regulatory Physician facing the ARMs

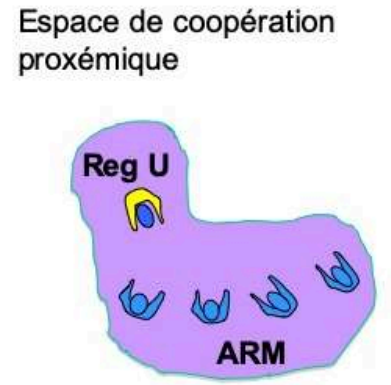

\begin{tabular}{|c|c|}
\hline Capacités & Effets \\
\hline $\begin{array}{l}\text { - Pouvoir avoir } \\
\text { une écoute de la } \\
\text { conversation de } \\
\text { l'ARM avec } \\
\text { Pappelant }\end{array}$ & $\begin{array}{l}\text { - anticiper et gagner du temps : } \\
\text { - de réception des infos du PARM } \\
\text { - en préparant le «questionnement» } \\
\text { avec l'appelant } \\
\text { - de décision d'envoyer une } \\
\text { ambulance } \\
\text { - ... }\end{array}$ \\
\hline $\begin{array}{l}\text { - Pouvoir être } \\
\text { interpellé par } \\
\text { PARM }\end{array}$ & $\begin{array}{l}\text { - anticiper et gagner du temps } \\
\text { - commentaires plus fournis que } \\
\text { sur le dossier } \\
\text { - arbitrage entre deux priorités (ne } \\
\text { pas se fier à l'ordre informatique } \\
\text { qui ne rend pas compte de la } \\
\text { priorité) }\end{array}$ \\
\hline
\end{tabular}

51 La figure 7 illustre l'intérêt de la proximité pour permettre au Med Rég U d'être en soutien à tout moment au médecin régulateur de ville.

Figure 7 : Médecin Régulateur d'Urgence à côté du médecin régulateur de ville.

Figure 7: Emergency Medical Regulator next to the City Medical Regulator

\begin{tabular}{|c|c|c|c|}
\hline \multicolumn{2}{|c|}{$\begin{array}{l}\text { Espace de coopération } \\
\text { proxémique }\end{array}$} & Capacités & Effets \\
\hline$\stackrel{\operatorname{Reg} u}{\rho}$ & Reg $v$ & $\begin{array}{l}\text { - Pouvoir avoir une } \\
\text { écoute de la } \\
\text { conversation du REG } \\
\text { Ville avec l'appelant }\end{array}$ & $\begin{array}{l}\text { - vérifier } \\
\text { - la cohérence du } \\
\text { dispatching ARM } \\
\text { - le caractère non urgent }\end{array}$ \\
\hline & & $\begin{array}{l}\text { - Pouvoir interpeller } \\
\text { le REG Ville }\end{array}$ & $\begin{array}{l}\text { - obtenir une explication liée } \\
\text { à sa compétence de } \\
\text { généraliste }\end{array}$ \\
\hline
\end{tabular}

\subsubsection{Les raisons d'un rapport de proximité de l'ARM avec les autres métiers de} la salle de régulation

La figure 8 montre que la capacité de l'ARM est plus importante du fait de pouvoir observer la disponibilité du médecin régulateur d'urgence, savoir s'il peut interférer dans son activité, l'alerter sur le niveau d'urgence d'un appel par des communications gestuelles et posturales dans son champ de vision. 
Figure 8 : ARM face au Médecin régulateur. Figure 8: ARM facing the Regulatory Physician

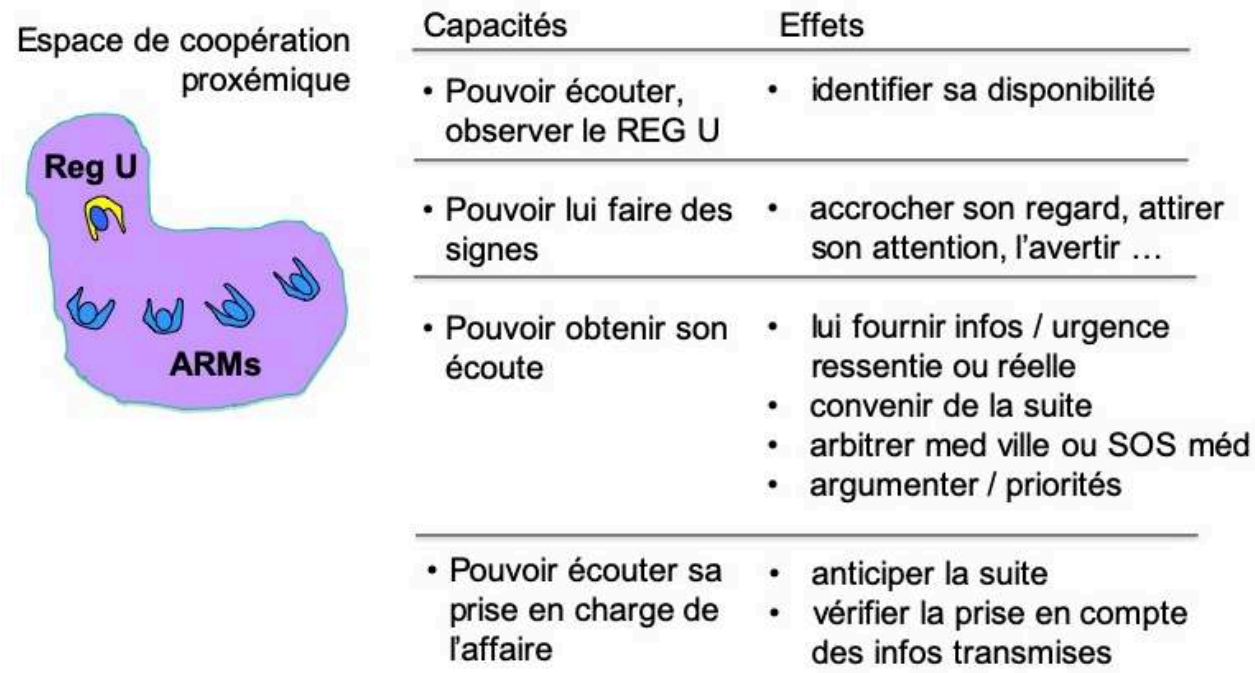

53 La figure 9 indique que cette proximité joue également en faveur d'un langage des gestes entre ARMs.

Figure 9 : ARM à côté de ses collègues.

Figure 9: ARM next to his colleagues

\begin{tabular}{|c|c|c|}
\hline \multirow{2}{*}{$\begin{array}{l}\text { Espace de coopération } \\
\text { proxémique }\end{array}$} & Capacités & Effets \\
\hline & $\begin{array}{l}\text { - Pouvoir écouter et } \\
\text { observer d'autres } \\
\text { ARM }\end{array}$ & $\begin{array}{l}\text { - Identifier et convenir } \\
\text { des situation urgentes } \\
\text { présentées au REG U } \\
\text { et en attente pour } \\
\text { arbitrage }\end{array}$ \\
\hline
\end{tabular}

\subsection{Repérage des aspects favorables et défavorables de la conception actuelle de la salle de régulation}

Même s'il est jugé facilitateur des communications, l'agencement dans l'ancienne salle de régulation présentait toutefois des inconvénients.

L'alignement de bureaux en deux marguerites et demi favorisait certes les communications à l'intérieur de l'une d'entre elles, mais les rendait moins souples et contraignantes d'une marguerite à l'autre. La figure 10 illustre les situations dos à dos et d'éloignement. Or, un ARM peut avoir besoin de communiquer avec un médecin régulateur situé à une autre extrémité de l'agencement. 
Figure 10 : Les contraintes du regroupement par 4 bureaux. Figure 10: The constraints of 4 desk groupings

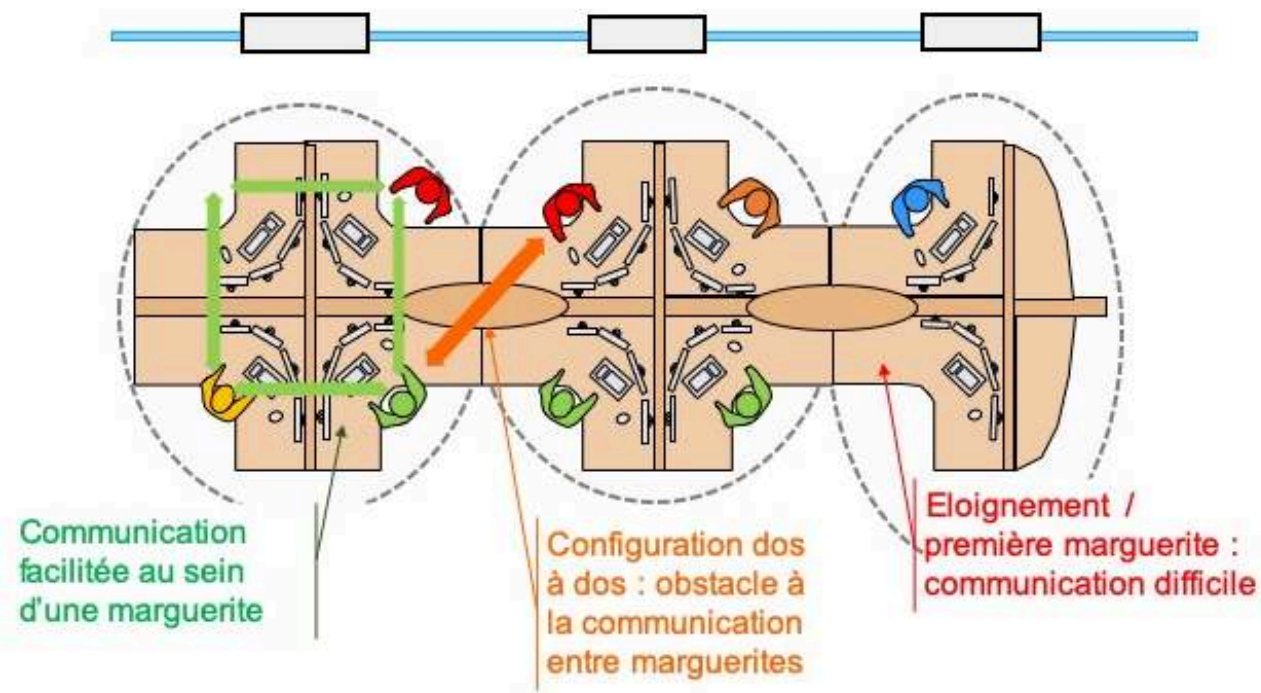

En fait, l'occupation des postes en marguerites et donc les distances entre collègues sont étroitement liées aux variations de l'effectif selon la tranche horaire dans la journée, le jour de la semaine et les évènements programmés (ex. un lendemain de réveillon...). Par exemple, en 2014 :

- la configuration dimanche matin mobilisait deux médecins régulateurs et quatre ARMs, avec une organisation en deux sous-pôles composés chacun d'un médecin régulateur et de deux ARMs ; ainsi, chaque sous-pôle pouvait investir une marguerite et bénéficier des proximités des postes;

- la configuration journée fonctionnait avec un effectif réduit à un ou deux médecins régulateurs et trois ARMs; dans le cas de la présence de deux médecins dépendants des appels collectés par les trois ARMs, le déploiement de l'équipe sur deux marguerites limitait les coopérations.

Le projet de déménagement offrait donc une opportunité de concevoir des agencements capables de présenter des proximités spatiales, quelle que soit la taille de l'équipe.

\section{Le dessin de ce que serait une configuration de postes « capacitante »}

Le même groupe de travail s'est enfin focalisé sur l'aménagement de la nouvelle salle de régulation. La surface et les murs étaient d'ores et déjà imposés par le maître d'ouvrage. Nous devions scénariser l'aménagement en intégrant :

- l'accueil des deux pôles déjà à l'œuvre - le pôle Urgence et le pôle Permanence des soins ambulatoires (PDSA) - qui gèrent les appels selon le degré d'urgence ;

- la variation de l'effectif de chacun de ces pôles et de sa composition selon le moment et le contexte ;

- la prévision d'un troisième pôle pour tenir compte de l'évolution régulière du nombre d'appels;

- la présence d'un poste pour le coordinateur ambulancier ; 
- l'injonction du maître d'œuvre d'indiquer rapidement l'emplacement des réservations de sortie de faisceaux électriques au sol avant le coulage de béton ;

- la vue sur l'extérieur en direction des passages d'entrée et sortie des ambulances afin d'actualiser l'état de son parc ;

et, aussi :

- les tableaux des capacités requises pour chaque métier et les rapports de proximités qu'ils suggèrent ;

- la tension entre les besoins d'un espace protecteur de l'environnement sonore lors de la conversation avec l'appelant et les besoins d'interagir avec cet environnement sonore ;

- les besoins particuliers et individualisés d'environnement lumineux ;

- les situations d'apprentissage.

En considérant les éléments intangibles et les situations d'actions caractéristiques ${ }^{6}$ dans processus de régulations, selon les contextes, telles qu'identifiées lors du diagnostic, le groupe de travail a fait les arbitrages suivants :

- la répartition des pôles dans le périmètre « imposé ».

L'objectif était de trouver le meilleur compromis pour installer concrètement, avec les contraintes environnementales, les différents collectifs de coopération et de traitement des différents contextes de prise en charge des appels d'urgence. La figure 11 rappelle ces collectifs réellement coopérants.

Figure 11 : Espaces coopératifs à protéger dans la future salle de régulation. Figure 11: Cooperative spaces to be protected in the future dispatch center

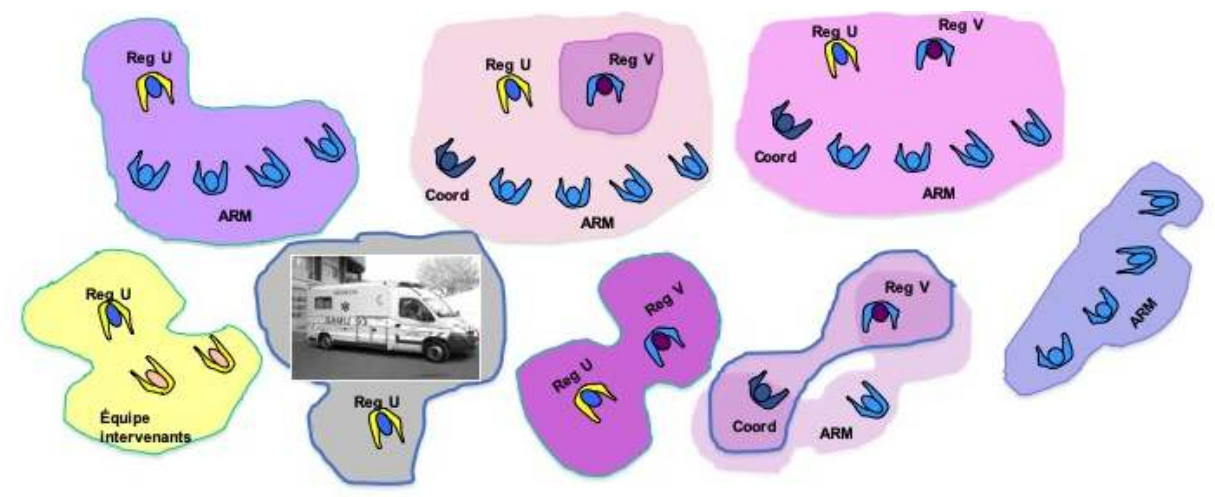

61 La figure 12 montre le compromis trouvé pour «placer » ces collectifs dans l'espace disponible et compte tenu des contraintes du bâtiment (emplacement des portes, des poteaux...). Puis les rangées de luminaires plafonds et parallèlement à la façade extérieure ont été positionnées de façon à obtenir des éclairements distincts et modulables selon la distance à la fenêtre. Le traitement sonore par l'acousticien s'est fait plus tard, lorsque le groupe de travail a su définitivement positionner les postes de travail de chacun des pôles sur le plan. En effet, l'emplacement des caissons que l'acousticien proposait devait impérativement s'établir en fonction d'un agencement définitif, pour disposer d'une localisation la plus précise des sources sonores émises par les travailleurs eux-mêmes. 
Figure 12 : Compromis pour agencer les 3 espaces coopératifs. Figure 12: Compromise for the layout of the 3 cooperative spaces

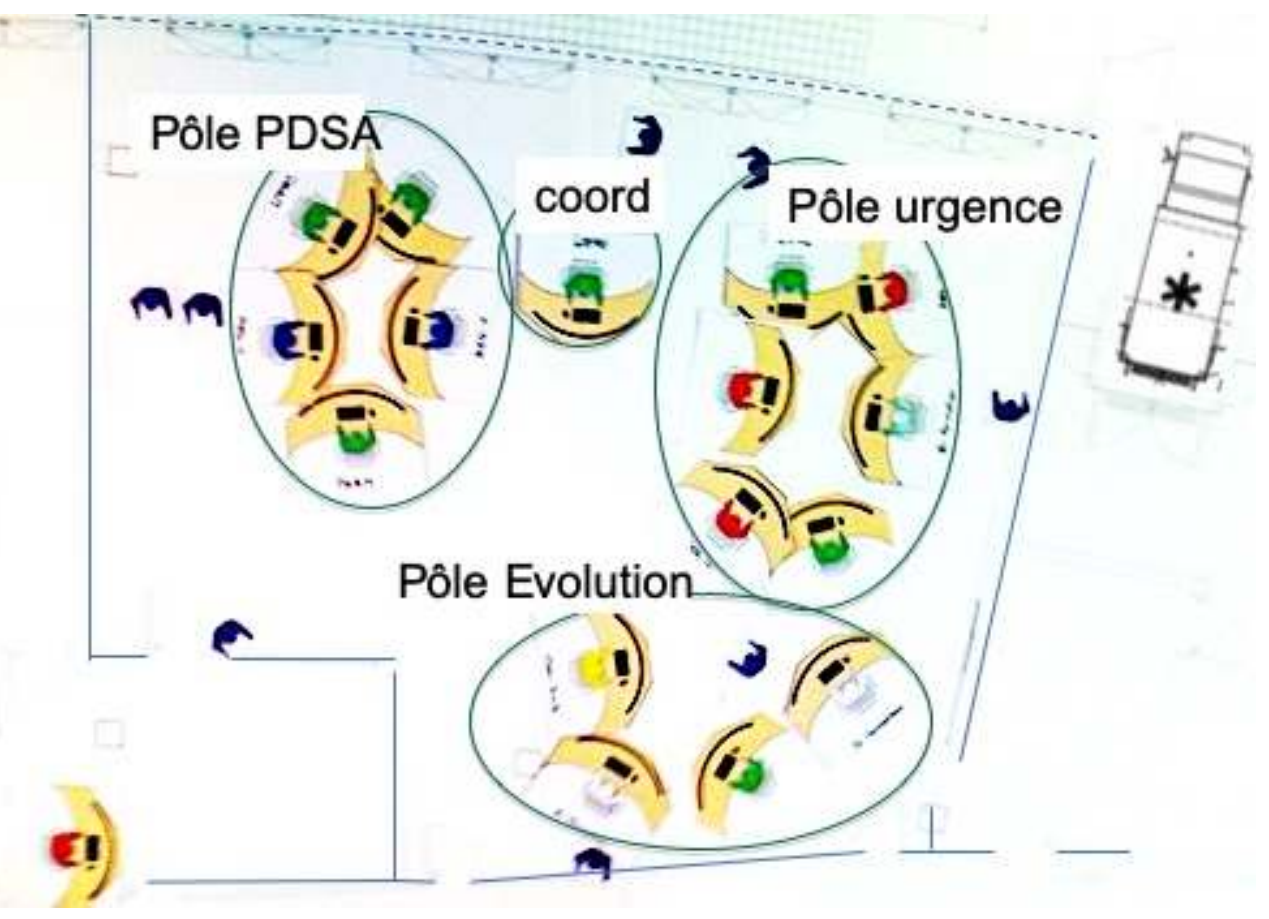

- le poste de travail incurvé : il présente l'avantage d'optimiser l'accès aux écrans en réduisant les rotations posturales; il répond en partie à la tension entre poste individuel et poste de pool ; le pool peut, à priori, se configurer en boucle de postes incurvés (voir Figure 13);

- une configuration en boucle de postes incurvés qui permet d'accueillir différents effectifs d'ARM et médecins régulateurs; le choix résulte de tests à l'échelle 1 (voir Figure 14) simulant les directions de regard, les comportements posturaux et les distances permettant de pouvoir écouter, se parler, s'alerter... 
Figure 13 : Forme de poste retenue et scénarios d'agencement. Figure 13: Type of desk selected and layout scenarios

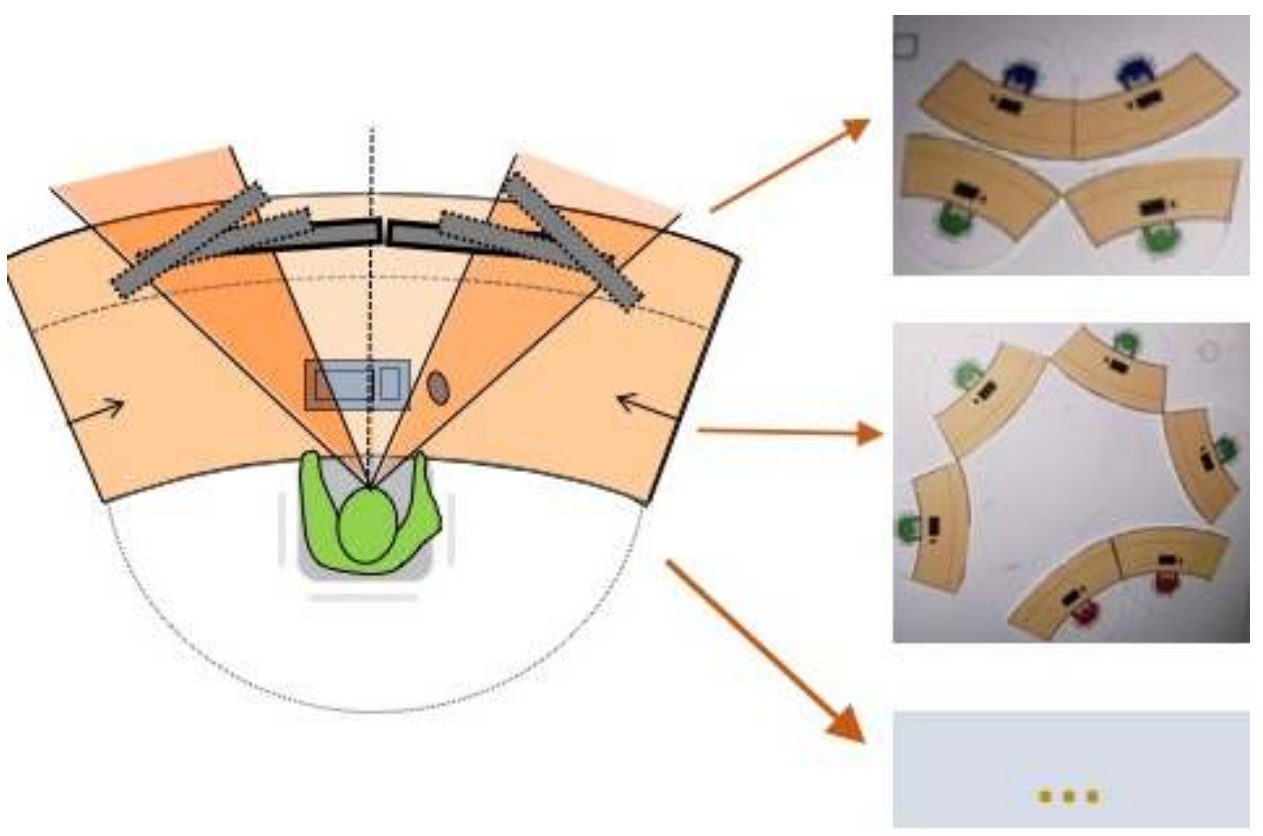

Figure 14 : Test d'agencement selon l'effectif présent.

Figure 14: Layout test according to the number of employees on site

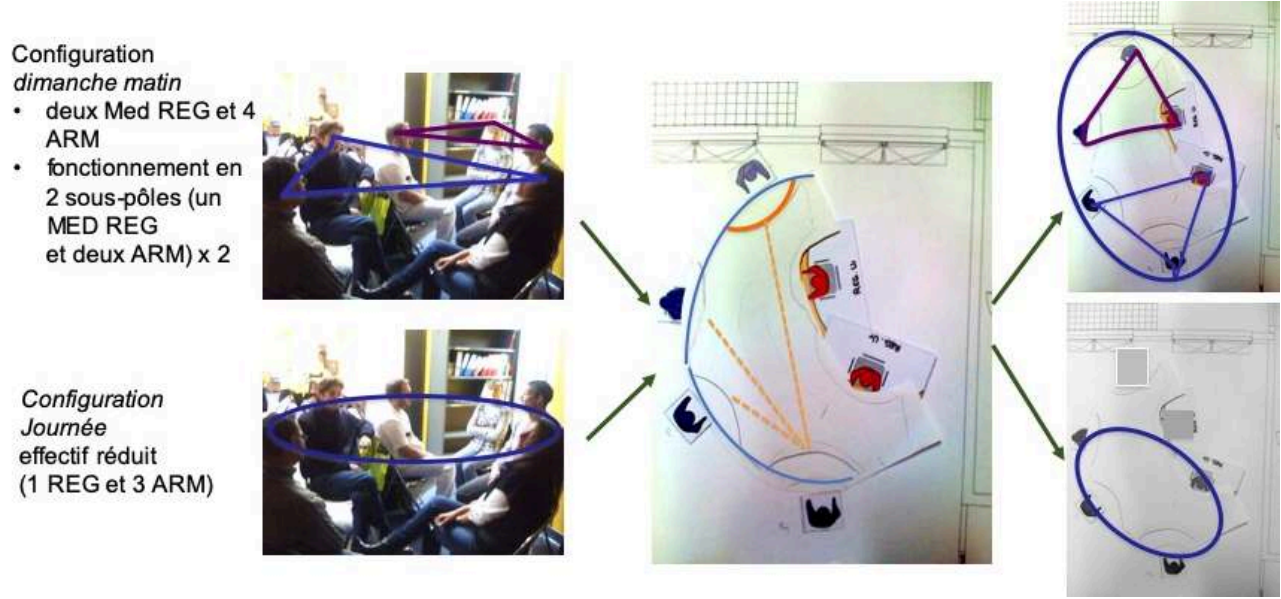

\section{Installation de la salle de régulation}

Le déménagement du SAMU et de sa salle de régulation a été effectué en octobre 2016, quatre années après la remise de notre cahier des charges à la maîtrise d'ouvrage.

L'installation finale correspondait aux conclusions du groupe de travail. Elle est au service de la transmission des informations par des communications verbales et orales entre ARMs et médecins régulateurs. La conception s'est finalement écartée d'un modèle qui accordait à ces communications un pouvoir perturbateur et a retenu le modèle accordant à celles-ci un pouvoir régulateur essentiel et nécessaire à l'atteinte des objectifs attendus d'un SAMU. 
64 La nouvelle salle de régulation permet aux ARMs, aux médecins régulateurs et médecins de ville de travailler dans le même espace de proximité et de pouvoir se voir et se parler.

L'implantation finale est très proche du scénario retenu. Globalement, la maîtrise d'œuvre a suivi les recommandations.

Deux ans après, le résultat est toujours apprécié par les utilisateurs. Peu après l'installation, aux premiers effets de l'usage, le groupe de travail s'est remobilisé pour modifier la répartition des postes autour du cercle du pôle urgence. D'une configuration avec deux médecins régulateurs côte à côte, chaque médecin s'est déplacé pour avoir une proximité immédiate, à droite et à gauche, avec les ARMs. Cette proximité permet une réduction des distances et optimisation des angles de vue propices à une amélioration de la communication orale et par le regard.

67 Des tableaux muraux et écrans géants complètent cette disposition pour suivre l'activité des SMUR du département et dans la perspective de situations de gestion de crise.

\section{Discussion}

Une démonstration est apportée, ici, de la confiance qu'il faut accorder aux pratiques d'usage des communications orales, gestuelles, posturales entre un (ou des) ARM(s) et un (ou des) médecin(s) régulateur(s). Elles interagissent certes pendant le processus obligatoire de saisie et transfert des premières informations que l'ARM doit collecter auprès de l'appelant et doit transmettre numériquement au médecin régulateur. Cette remarque correspond aux conclusions du travail de recherche mené avec l'Université Paris Est Créteil (Mouchet \& Bertrand, 2018)

69 Ces interactions se sont montrées, dans tous les cas observés, de nature à éviter des ruptures de flux dans le traitement des appels, des attentes problématiques et, en conséquence, à viser la plus grande réactivité et fiabilité.

70 L'analyse a montré qu'elles n'étaient pas "inconsidérées ", c'est-à-dire réalisées "n'importe comment ", de façon désordonnée, sur une simple impulsion individuelle. Elles sont toutes issues de conventions entre les acteurs de la salle de régulation, construites collectivement, appliquées sous l'œil et l'écoute de tous et évaluées par euxmêmes. La nature des gestes et postures observés rend compte d'un langage commun (Leplat, 2013). Une posture redressée, une posture debout, une variation d'intensité sonore, un silence prolongé, un signe de la main... mais aussi une tonalité et un niveau sonore différents dans la conversation... visent à faire comprendre qu'il y a un contexte particulier qui nécessite un mode de prise en charge particulier.

71 Lors de notre visite de la nouvelle salle de régulation, tous vantaient la qualité du traitement acoustique réalisé. Le bruit, pourtant résultant de nombreuses communications téléphoniques et de proximités, est atténué. Mais en plus de la raison technique, il $\mathrm{y}$ a les règles autour $\mathrm{du}$ son. Lorsque le traitement de l'appel se passe bien (pas de liste d'attente, pas d'urgence en attente, pas de contextes particuliers...), le niveau sonore émis par les conversations est bas, même s'il est entendable. Ceci permet de signaler plus facilement le besoin de communication rapide avec le médecin régulateur par un simple différentiel de niveau sonore dans la voix. C'est une convention entre eux. 

du compagnonnage. Nous avons observé aussi la manière d'un tuteur de signifier clairement au nouveau que, pour tel contexte de l'appel, il y a nécessité d'interférer directement et, en prenant la main, lui indique les règles (par ex. changer la nature de sa position au poste, élever le son de sa voix, identifier le niveau d'engagement du médecin régulateur dans son traitement en cours, identifier s'il a perçu son alerte, vérifier que d'autres ARMs ne sont pas dans la même situation, éventuellement négocier avec eux des priorités...).

Le témoignage que nous avons rapporté de notre intervention peut nous laisser penser que le drame que nous rappelons dans l'introduction est très spécifique dans la mesure où, à l'instant où il se produisait, probablement qu'aucune capacité d'écouter, intervenir, contrôler, aider et finalement interrompre le processus dramatique en cours... n'était au rendez-vous.

Dans une perspective d'évolution des SAMU, ce serait donc les espaces proxémiques et coopératifs qu'il faudrait promouvoir et consolider dans la conception de l'espace, dans les équipements, dans la formation, par le management.

Encore faudrait-il s'assurer que les décisions qui seraient destinées à rassurer l'opinion publique ne soient pas, pour le coup, «inconsidérées». Il ne faudrait pas que les procédures de traitement des appels soient standardisées au moyen d'outils numériques, des sortes de progiciels de gestion intégrés, qui seraient présentées comme des remparts à l'erreur humaine (Bourgeois \& Vanpoulle, 2018). Leurs promoteurs feraient, pour le coup, l'erreur de réduire l'appel à un objet isolable de l'ensemble des contextes différents, particuliers, surprenants, évolutifs... qui justifient justement le recours à des communications et régulations coopératives adaptées à la situation. Ils feraient l'erreur d'empêcher le travail d'explicitation et d'évaluation des pratiques individuelles et collectives qui s'expriment justement lors des communications, sous la supervision de tous (Mouchet \& Bertrand, 2018).

\section{BIBLIOGRAPHIE}

Benchekroun, T.H. (2000). Les espaces de coopération proxémiques. In T.H. Benchekroun, \& Weill-Fassina, A. (Eds.), Le travail collectif (pp. 35-54). Toulouse : Octarès.

Bertrand, C., Ammirati, C, Lecarpentier, E., Boyer, C., \& Marty, J. (2018). Les défis sociétaux et professionnels en régulation médicale : intérêt d'un nouveau regard sur le travail au SAMU. In Mouchet, A. \& Bertrand, C. (Eds.). Décider en Urgence au Sami centre 15. (pp. 21-36). Toulouse : Octarès.

Bourgeois, F., \& Hubault, F. (2013). L'activité, ressource pour le développement de l'organisation du travail. In P. Falzon (Ed.), L'ergonomie constructive (pp. 89-102). Paris : PUF.

Bourgeois, F., \& Vanpoulle, C. (2018). Réorganiser les centres d'appels d'urgence. Le Monde Eco du 30 mai 2018, https://mobile.lemonde.fr/idees/article/2018/05/29/samu-ne-pas-entraver-lescapacites-d-initiative-et-la-creativite-des-salaries_5306398_3232.html ? xtref = 
Caroly, S., \& Barcellini, F. (2013). Le développement de l'activité collective. In P. Falzon (Ed.). L'ergonomie constructive (pp. 33-46). Paris : PUF.

Clot, Y. (2008). Travail et pouvoir d'agir. Paris : PUF.

Daniellou, F. (2004). L'ergonomie dans la conduite de projets de conception de systèmes de travail. In P. Falzon (Ed.), Ergonomie (pp. 359-373), Paris : PUF.

Darses, F., \& Falzon, P. (1994). La conception collective : une approche de l'ergonomie cognitive. Séminaire GDR CNRS FROG, Toulouse. http://www.minnd.fr/wp-content/uploads/2015/03/ concep_collect_96-Darses-Falzon.pdf

Falzon, P. (2005). Ergonomie, conception, développement. Laboratoire d'ergonomie du CNAMParis. $40^{e}$ Congrès SELF, St Denis de la Réunion http://ergonomie.cnam.fr/equipe/falzon/ articles_pf/ergo_concep_develop_05.pdf

Leplat, J. (2001). La gestion des communications par le contexte. Pistes, 3(1). www.unites.uqam.ca/ pistes :v3n1

Leplat, J. (2013). Les gestes dans l'activité en situation de travail. Aperçu de quelques problèmes d'analyse. Pistes, 15(1). https://journals.openedition.org/pistes/2951

Leyreloup, M. (2008). Compte rendu de lecture. In A. Borzeix \& B. Fraenkel (Eds.), Langage et Travail, Communication, cognition, action. Mots, les langages politiques (en ligne) 69/2002. http://journals.openedition.org/mots/10793

Marc, J., \& Amalberti, R. (2002). Contribution individuelle à la sécurité du collectif : exemple de la régulation du SAMU. Le Travail Humain, 65, 217-242.

Maggi, B. (1996). La régulation du processus d'action de travail. In P. Cazamian, F. Hubault, \& M. Moulin (Eds.), Traité d'ergonomie (pp. 637-659). Toulouse : Octarès.

Mayen, P. (2000). Pour une intégration du cadre pragmatique d'analyse des interactions verbales au cadre d'analyse ergonomique des situations de travail. $35^{e}$ Congrès de la SELF, Toulouse, https:// ergonomie-self.org/wp-content/uploads/2016/01/toulouse-2000-congres-self-1-analysercommunication.pdf

Mouchet, A., \& Bertrand, C. (Eds.) (2018). Décider en Urgence au Sami centre 15. Toulouse : Octarès Morvan, E., de Terssac, G., \& Peytavin, A. (2000). Communiquer pour assurer la sécurité dans les systèmes distribués. $35^{e}$ Congrès de la SELF, Toulouse, https://ergonomie-self.org/wp-content/ uploads/2016/01/toulouse-2000-congres-self-11-collectifs-travail.pdf

Oudet, S.F. (2012). Concevoir des environnements de travail capacitants : l'exemple d'un réseau réciproque d'échanges des savoirs. Revue Française de Sciences Sociales, 119, 7-27.

Vanpoulle, C. Bourgeois, F., Ammirati, C., Boyer, C., Amsallem, C., \& Decagny, P. (2016). Conception d'une salle de régulation des Urgences : exigences de fiabilité et besoins des futurs utilisateurs sont-ils compatibles ? $52^{e}$ Congrès de la SELF, Toulouse, https://ergonomie-self.org/ auteur/vanpoulle-c/

\section{NOTES}

1. "Enquête sur les circonstances ayant conduit au décès d'une jeune patiente suite aux sollicitations du SAMU de Strasbourg le 29 décembre 2017 », rapport définitif, juin 2018 (www.igas.gouv.fr/IMG/pdf/2018-061R-Rapport_definitif.pdf) 
2. Synthèse des recommandations de bonne pratique pour la prise en charge d'un appel de demande de soins non programmés dans le cadre de la régulation médicale

3. Arrêté du 4 juin 2013 relatif à la formation d'adaptation à l'emploi des membres du corps des assistants médico-administratifs de la fonction publique hospitalière (branche assistance en régulation médicale)

4. Pouvoir d'agir (Clot, 2008) : il mobilise chez le sujet son pouvoir, son vouloir «faire» et "prendre les moyens de le faire »

5. Pouvoir il mobilise un environnement (contexte, organisation du travail, mode de management...) qui donne les moyens de la prise de responsabilité, de la prise de risques.

6. Dans le domaine de l'ergonomie, une situation d'action caractéristique (SAC) est une famille de situations que les opérateurs ou utilisateurs ont à contrôler. Elle peut être attachée à une étape d'un processus de production ou de service (Daniellou, 2004).

\section{RÉSUMÉS}

À l'occasion d'une délocalisation dans le nouveau $\mathrm{CHU}$, la conception de la salle de régulation des Appels du SAMU de la Somme a fait l'objet d'une réflexion opposant, d'une part, une approche théorique de la fiabilité qui s'est imposée dans les premières esquisses et qui visait à réduire, voire empêcher les communications orales et, d'autre part, une approche pragmatique et empirique des utilisateurs qui soutenaient l'utilisation des communications comportementales et orales dans le traitement des appels. Les acteurs de cette salle de régulation se sont invités dans la conduite du projet pour y intégrer leur expertise. En écho au drame survenu lors de la prise en charge inadaptée d'une appelante dans un SAMU, cette communication rend compte de l'importance de la conception de la salle de régulation dans sa capacité à favoriser des coopérations proxémiques qui sont de nature à superviser et anticiper d'éventuelles dérives solitaires et individuelles.

When it was relocated to the new university hospital, the design of the SAMU dispatch center in France (Somme region) France became an object of debate regarding: on the one hand, a theoretical approach to reliability that emerged at the time of its creation and which aimed to reduce or even prevent oral communications and, on the other hand, a pragmatic and empirical approach by users who supported behavioural and oral communications in the processing of calls. Dispatch center staff set out to lead the redesign project and integrate their expertise.

In response to the tragedy that occurred after the inappropriate treatment of one caller to a SAMU center, this presentation reflects on the importance of the design of the dispatch room, and its ability to foster proxemic cooperation capable of anticipating possible individual misconduct.

\section{INDEX}

Mots-clés : conception, communications, espace de travail, coopération, environnement capacitant, fiabilité

Keywords : conception, communications, workspace, cooperation, enabling environment, reliability 


\section{AUTEURS}

\section{FABRICE BOURGEOIS}

Ergonome consultant à Concilio ergonomie, chargé de cours à l'université Paris ouest Nanterre, f.bourgeois@concilio-ergonomie.fr

\section{CHRISTOPHE VANPOULLE}

Ergonome consultant à Concilio ergonomie, chargé de cours à l'université Jules Verne Picardie, c.vanpoulle@concilio-ergonomie.fr

\section{CHRISTINE AMMIRATI}

Chef du pôle médecine d'urgence, CHU Amiens, 80054 Amiens Cedex 1, Christine.ammirati@chuamiens.fr 